\title{
Feasibility of a prehabilitation program before major abdominal surgery: a pilot prospective study
}

Journal of International Medical Research 49(II) I-II

(C) The Author(s) 2021

Article reuse guidelines: sagepub.com/journals-permissions DOI: I0.1 I77/030006052II060I96 journals.sagepub.com/home/imr

@SAGE

\author{
David Martin' (D), Cyril Besson ${ }^{2,5}$, \\ Basile Pache' ${ }^{(D,}$, Anna Michel ${ }^{3}$, \\ Sandrine Geinoz', \\ Vincent Gremeaux-Bader ${ }^{2,5}$, Anna Larcinese ${ }^{4}$, \\ Charles Benaim ${ }^{6}$, Bengt Kayser ${ }^{5}$ (D), \\ Nicolas Demartines' and Martin Hübner'
}

\begin{abstract} formance and outcomes after major abdominal surgery. were evaluated.

\footnotetext{
'Department of Visceral Surgery, University Hospital CHUV and University of Lausanne (UNIL), Lausanne, Switzerland

${ }^{2}$ Department of Sports Medicine, Swiss Olympic Medical Center, University Hospital CHUV and University of Lausanne (UNIL), Lausanne, Switzerland

${ }^{3}$ Faculty of Biology and Medicine, University of Lausanne (UNIL), Lausanne, Switzerland

${ }^{4}$ Department of Physiotherapy, University Hospital CHUV, Lausanne, Switzerland
}

${ }^{5}$ Institute of Sport Sciences, University of Lausanne (UNIL), Lausanne, Switzerland
\end{abstract}

Objective: To assess the feasibility of a prehabilitation program and its effects on physical per-

Methods: In this prospective pilot study, patients underwent prehabilitation involving three training sessions per week for 3 weeks preoperatively. The feasibility of delivering the intervention was assessed based on recruitment and adherence to the program. Its impacts on fitness (oxygen uptake $\left(\mathrm{VO}_{2}\right)$ ) and physical performance (Timed Up and Go Test, 6-Minute Walk Test)

Results: From May 2017 to January 2020, 980 patients were identified and 44 (4.5\%) were invited to participate. The main obstacles to patient recruitment were insufficient time $(<3$ weeks) prior to scheduled surgery $(n=276,28 \%)$ and screening failure $(n=312,32 \%)$. Of the 44 patients, 24 (55\%) declined to participate, and 20 (23\%) were included. Of these,

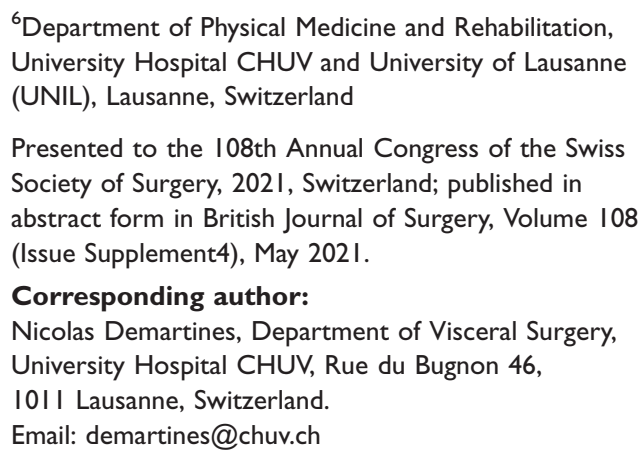

${ }^{6}$ Department of Physical Medicine and Rehabilitation, University Hospital CHUV and University of Lausanne (UNIL), Lausanne, Switzerland

Presented to the 108th Annual Congress of the Swiss Society of Surgery, 202I, Switzerland; published in abstract form in British Journal of Surgery, Volume 108 (Issue Supplement4), May 202I.

\section{Corresponding author:}

Nicolas Demartines, Department of Visceral Surgery, University Hospital CHUV, Rue du Bugnon 46,

I0II Lausanne, Switzerland.

Email: demartines@chuv.ch 
six $(30 \%)$ were not adherent to the program. Among the remaining 14 patients, $\mathrm{VO}_{2}$ at ventilatory threshold significantly increased from 9.7 to $10.9 \mathrm{~mL} / \mathrm{min} / \mathrm{kg}$. No significant difference in physical performance was observed before and after prehabilitation.

Conclusion: Although prehabilitation seemed to have positive effects on exercise capacity, logistic and patient-related difficulties were encountered. The program is not feasible in its current form for all-comers.

\section{Keywords}

Prehabilitation, major abdominal surgery, physical activity, outcome, recruitment, adherence, pilot study

Date received: 4 August 202I; accepted: 28 October 2021

\section{Introduction}

Physical fitness is an important predictor of postoperative recovery and outcomes after abdominal surgery. ${ }^{1,2}$ Preoperative physical exercise training, termed prehabilitation, complements the traditional models of recovery by initiating the recovery process preoperatively. ${ }^{3,4}$ Improvement of physical fitness by means of prehabilitation can speed up recovery after surgery. ${ }^{5-7}$ Results of a systematic review suggested that prehabilitation can effectively reduce postoperative complication rates and shorten the hospital stay. ${ }^{8}$ Other studies showed that in patients undergoing major abdominal surgery, preoperative exercise therapy was associated with improved physical fitness, resulting in fewer complications and faster convalescence. ${ }^{9,10}$

Several non-randomized studies have suggested that $\geq 6$ weeks of supervised exercise programs can improve physical capacity. ${ }^{11-13}$ However, a 6-week delay before surgery is not always feasible, especially in patients with malignant disease. ${ }^{14}$ Given the short time window available for prehabilitation, any strategies that are applied should be as efficient as possible to achieve worthwhile changes within this preoperative timeframe. ${ }^{15,16}$
In preparation for a future randomized clinical trial (RCT), the present study was performed to assess the feasibility of a 3-week supervised preoperative prehabilitation program and its effects on physical performance and clinical outcomes after major abdominal surgery.

\section{Patients and methods}

\section{Study design and participants}

This prospective pilot study was performed in preparation for an RCT on prehabilitation before major abdominal surgery at the Department of Visceral Surgery, University Hospital CHUV, Lausanne, Switzerland, a tertiary academic hospital. The study lasted from May 2017 to January 2020. Major abdominal surgery was defined as any esophageal, gastric, hepatic, pancreatic, intestinal, or colorectal surgery for benign or malignant disease, either open or laparoscopic and lasting more than 2 hours. The exclusion criteria were metabolic surgery, organ transplantation, coronary artery disease (stage $\geq$ III according to the Canadian Cardiovascular Society), ${ }^{17}$ heart failure (stage $\geq$ III according to the New York Heart Association), ${ }^{18}$ uncontrolled cardiac arrhythmias, chronic obstructive 
pulmonary disease (stage $\geq$ III according to the Global Initiative for Chronic Obstructive Lung Disease), ${ }^{19}$ any other surgery within 6 weeks prior to enrollment, and a delay of $<3$ weeks between consultation and surgery. Patients were enrolled at the preadmission consultation by the operating surgeons, and all benefited from the Enhanced Recovery After Surgery program. ${ }^{20-22}$

\section{Outcomes}

The primary outcome of the study was the feasibility of delivering a prehabilitation program based on recruitment and adherence. Recruitment was determined by the number of eligible participants included, and exercise adherence was defined as the percentage of patients who completed the prehabilitation (at least eight exercise sessions of the nine proposed). This pilot trial would be deemed feasible if $\geq 70 \%$ of eligible participants were successfully recruited and $\geq 80 \%$ of the recruited participants completed the prehabilitation, as previously described. ${ }^{23}$

The secondary outcomes were the impact of prehabilitation on 30-day postoperative morbidity using the Comprehensive Complication Index $(\mathrm{CCI})^{24}$ and the impact of prehabilitation on the overall rate of complications according to the Clavien classification. ${ }^{25}$ The CCI summarizes all postoperative complications and is computed on the basis of tabulated complications according to the Clavien classification. Other outcomes included length of stay (LOS), exercise capacity (maximum oxygen uptake $\left(\mathrm{VO}_{2} \max \right)$ ), four measures of physical activity performance (cardiopulmonary exercise testing (CPET), Timed Up and Go Test (TUGT), 6-Minute Walk Test (6-MWT), handgrip strength test (dynamometer)), and quality of life (European Organization for Research and Treatment of Cancer Quality of Life Questionnaire (EORTC QLQ-C30)). ${ }^{26}$ Patients performed the four measures of physical performance and the QLQ-C30 before and after prehabilitation. CPET was performed on an electromagnetically braked cycle ergometer using an incremental protocol until volitional termination, as previously described. ${ }^{27}$ Ventilation, gas exchange, pulse rate, 12-lead electrocardiography, noninvasive blood pressure, and pulse oximetry were monitored throughout the test. Derived variables included $\mathrm{VO}_{2}$ at ventilatory threshold $\left(\mathrm{VO}_{2} \mathrm{AT}, \mathrm{V}\right.$-slope method) and at peak. The TUGT and 6-MWT were performed as previously described. ${ }^{28}$ For handgrip dynamometry, participants stood in an upright position with their hands positioned by their sides, holding the dynamometer in the dominant hand, and squeezed for 3 seconds before relaxing (three repetitions). ${ }^{15}$ The EORTC QLQ-C30 is an internationally validated cancer-specific questionnaire. $^{29}$ The scores range from 0 to 100 after linear transformation of the raw scores. A high score for a functional scale represents a high level of functioning (healthier), whereas a high score for a symptom scale represents a high level of symptoms/problems. ${ }^{30}$ The study case report form is available as supplementary material.

\section{Prehabilitation program}

The prehabilitation program involved three training sessions per week for 3 weeks preoperatively (nine sessions). Exercises were based on high-intensity interval training (HIIT) and carried out on a braked cycle ergometer. ${ }^{16}$ Each session consisted of a 5-minute warm-up (at 50\% of peak CPET power reached (Wpeak)) followed by two 10 -minute series of 15-s high-intensity intervals at $100 \%$ Wpeak interspersed by a 15 -s active recovery period $(30 \%)$ and a 4-minute rest period between the two series. The session ended with a cool-down 
period (5-minute active recovery period at $30 \%$ Wpeak).

\section{Pilot study}

An upfront pilot study including 20 patients (prehabilitation only) was requested by the institutional review board to assess feasibility of recruitment and adherence to the intervention.

\section{Statistical analysis}

Continuous variables are presented as mean \pm standard deviation or median (interquartile range) and were compared with the Mann-Whitney U test or Student's t test according to their distribution (ShapiroWilk test). Categorial variables are presented as frequency (percentage) and were compared with Pearson's chi-square test or Fisher's exact test as appropriate. Linear relationships between $\mathrm{VO}_{2}$ peak, $\mathrm{CCI}$, and LOS were quantified by Pearson correlation. A p-value of $\leq 0.05$ was considered statistically significant, and all tests were two-sided. Analysis was performed using IBM SPSS Statistics for Windows, Version 25.0 (IBM Corp., Armonk, NY, USA). Missing data were omitted based on the available case analysis (pairwise).

\section{Ethics}

The study protocol $n^{\circ} 469 / 15$ was approved by the Institutional Review Board (Commission cantonale d'éthique de la recherche sur l'être humain, CER-VD, Lausanne, Switzerland) on 29 March 2016 (approval number: 2016-00896). All patients provided written informed consent, and their details were de-identified. The study was carried out in line with the STROBE statement ${ }^{31}$ and registered at https://clinicaltrials.gov/ (NCT02953119).

\section{Results}

\section{Feasibility}

During the study period, 980 patients were identified, of whom 586 met the inclusion criteria. Forty-four $(4.5 \%)$ patients were invited to participate. The other patients were not invited, mainly because of insufficient time $(<3$ weeks) available prior to scheduled surgery $(n=276,28 \%)$ or screening failure ( $n=312,32 \%)$ (Figure 1). Of the 44 approached patients, 24 (55\%) declined to participate and $20(45 \%)$ were finally included. Among those included, $14(70 \%)$ remained adherent to the prehabilitation program. Reasons for nonadherence were a contraindication for exercise training evidenced during CPET $(n=3)$, discontinuation of the program by the patient because of lack of motivation $(n=2)$, and modification of the planned operating date $(n=1)$. Of the three patients for whom the program was interrupted for medical reasons, one developed vagal discomfort with dizziness and nausea, and the other two presented electrocardiographic changes without symptoms during CPET. Subsequent investigations with a cardiac magnetic resonance imaging stress test were unremarkable, and no sequelae were noted.

\section{Clinical outcomes}

Patient demographics and surgical details are displayed in Table 1 . Six (43\%) of the 14 patients developed complications (nine complications occurred among these 6 patients). Most complications were minor (Clavien grade I-IIIa, $\mathrm{n}=7,78 \%$ ), although major complications also occurred (Clavien grade IIIb, $\mathrm{n}=2,22 \%$ ). The mean CCI of patients with complications was $13.7 \pm 18.5$, and one patient had to be readmitted. The median LOS was 4 days (interquartile range, 3-5 days). 


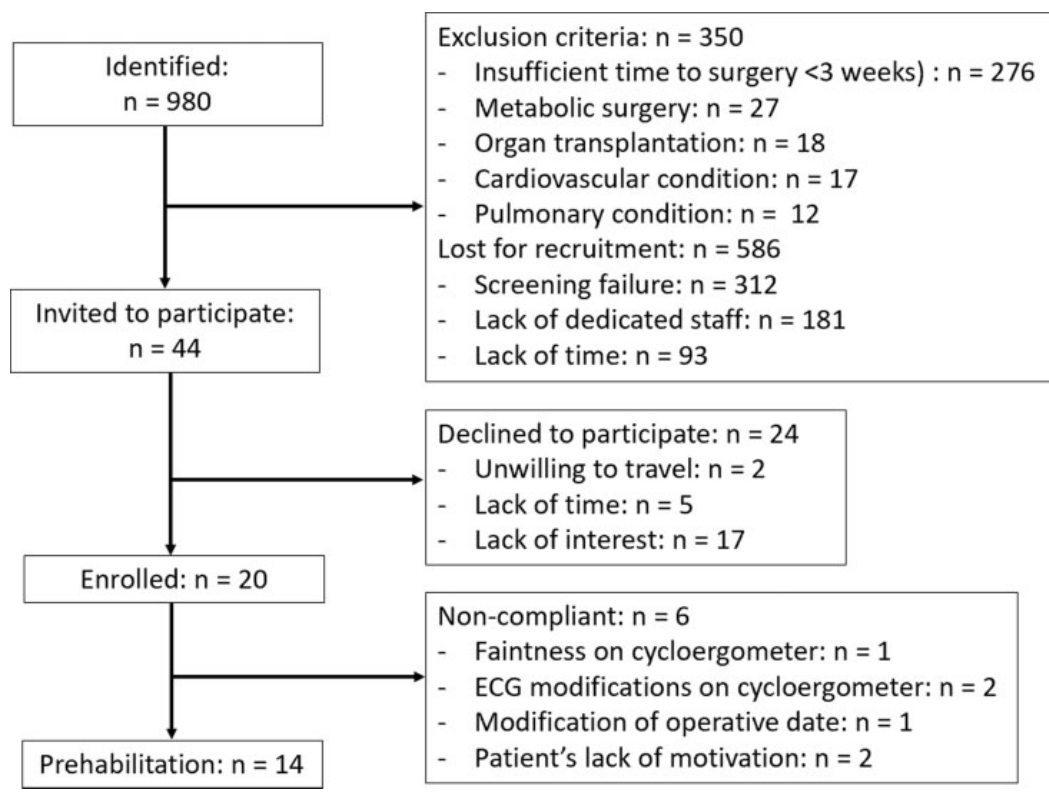

Figure I. Study flowchart. ECG, electrocardiographic.

\section{Physical functioning}

The mean prehabilitation time was $28 \pm 6$ days, and the second CPET was performed $5 \pm 2$ days before surgery. The mean $\mathrm{VO}_{2}$ AT increased from 9.7 to $10.9 \mathrm{~mL} /$ minute/ $\mathrm{kg}$ after prehabilitation $(\mathrm{p}=0.009)$, and the mean $\mathrm{VO}_{2}$ max increased from 18.6 to 21.0 $\mathrm{mL} /$ minute $/ \mathrm{kg}(\mathrm{p}=0.006)$. Twelve $(86 \%)$ of the 14 patients exhibited improvement in their $\mathrm{VO}_{2}$ AT. There was no significant difference in handgrip dynamometry before and after prehabilitation ( 31.7 vs. $33.0 \mathrm{~kg})$. The results of the TUGT and 6-MWT before and after prehabilitation are shown in Figure 2; no significant differences were found. No correlations were observed between the change in $\mathrm{VO}_{2}$ max after prehabilitation and the postoperative CCI $(r=-0.133)$ and $\operatorname{LOS}(r=-0.94)$.

\section{Quality of life}

Changes in quality of life after prehabilitation are displayed in Figure 3. Physical functioning was the only element that significantly improved $(82.5$ vs. 93.4, $\mathrm{p}=0.040)$.

\section{Discussion}

This pilot study emphasized the practical difficulties in implementing a prehabilitation program for all-comers before major abdominal surgery. The program is not feasible in its current form. The organization failed, even in a department with broad experience in perioperative medicine. A too-short time window $(<3$ weeks) prior to surgery and a lack of commitment from surgical staff and patients were major barriers to implementation. The consecutively planned randomized study was therefore not performed.

Only $5 \%$ of potentially eligible patients were actually approached during the recruitment phase, and among these, 45\% were recruited for inclusion. These rates are low. Another feasibility study of a 
Table I. Patient demographics and surgical details.

\begin{tabular}{|c|c|}
\hline & Overall $(n=14)$ \\
\hline Age, years & $64 \pm 13.9$ \\
\hline Body mass index, $\mathrm{kg} / \mathrm{m}^{2}$ & $28.4 \pm 5.9$ \\
\hline Sex, male:female & $8: 6$ \\
\hline $\begin{array}{l}\text { Alcohol consumption } \\
\qquad>\text { I unit/day })\end{array}$ & $7(50)$ \\
\hline $\begin{array}{l}\text { Active smoking } \\
\text { (daily or occasionally) }\end{array}$ & $5(36)$ \\
\hline Cardiovascular disease & I (7) \\
\hline Chronic pulmonary disease & $0(0)$ \\
\hline Diabetes & $4(29)$ \\
\hline \multicolumn{2}{|l|}{ ASA score } \\
\hline$(\mathrm{I}-\mathrm{II})$ & II (79) \\
\hline (III-IV) & $3(21)$ \\
\hline \multicolumn{2}{|l|}{ Reason for referral } \\
\hline Gastrointestinal bleeding & $3(2 I)$ \\
\hline Cancer screening & $4(29)$ \\
\hline \multicolumn{2}{|c|}{ Metastases on extension workup3 (2I) } \\
\hline Other & $4(29)$ \\
\hline \multicolumn{2}{|l|}{ Surgical procedure } \\
\hline Colorectal & $9(64)$ \\
\hline Liver & $3(2 I)$ \\
\hline Other & $2(15)$ \\
\hline Malignancy & $7(50)$ \\
\hline Laparoscopic approach & $9(64)$ \\
\hline
\end{tabular}

Data are presented as mean \pm standard deviation, $n$, or n (\%).

ASA, American Society of Anesthesiologists.

prehabilitation program in patients undergoing elective colorectal surgery showed a similarly low recruitment rate $(18 \%)$, and $58 \%$ of the initially eligible patients were approached. ${ }^{15}$ One reason for low recruitment in the present study was an insufficient window of available time prior to scheduled surgery (28\% of patients). However, Northgraves et al. ${ }^{15}$ reported a slightly higher rate of up to $40 \%$. In the United Kingdom, the National Health Service currently recommends that treatment in patients with cancer should be initiated within 31 days of the decision to treat, providing only a short window of opportunity to implement prehabilitation. ${ }^{32}$ A systematic review showed that the duration of preoperative exercise programs ranged from 21 to 74 days. ${ }^{33}$ Thus, any strategies applied should be efficient enough to achieve physical capacity changes within this relatively short preoperative timeframe. The HIIT-based models chosen in this study apply particularly well to these criteria. ${ }^{11,14-16}$

Other barriers to recruitment in the present study were screening failure and lack of allocated staff, even before meeting the patient. The availability of a dedicated research nurse may have improved the recruitment rate, as suggested by others. ${ }^{15}$ Still, once approached, $55 \%$ of patients refused to participate because of lack of time and interest and, to a lesser extent, unwillingness to travel. Another study showed that like in the present study, common reasons for non-participation in such training included insufficient free time, being unable to travel, and having other personal commitments, especially in the professionally active patient population. ${ }^{15}$ These findings highlight the fact that not only organizational and logistical aspects but also broad information, motivations, and beliefs of both caregivers and patients must be taken into consideration. The cost aspect is probably also important because reimbursement for this novel approach with limited evidence is not warranted in all countries. However, a systematic review showed that no studies addressed the economic implications or cost-effectiveness of prehabilitation programs, which could be either a critical barrier to implementation in routine clinical practice or an argument in favor of prehabilitation. $^{34}$

Once included, $70 \%$ of patients in the present study remained adherent to the prehabilitation program. The main reasons for non-adherence were physiological problems during CPET and discontinuation of the program by the patient because of lack of motivation, which represented an 


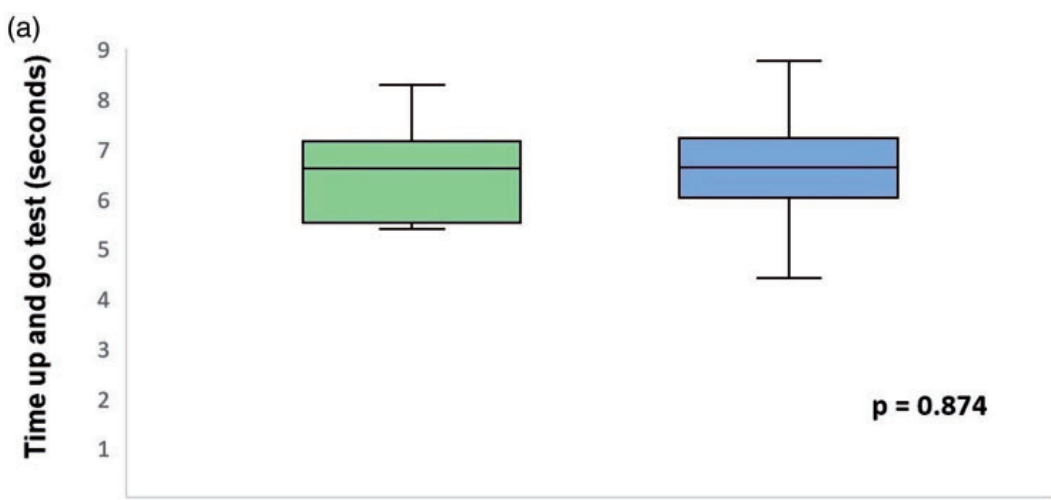

Before prehabilitation

After prehabilitation

(b)
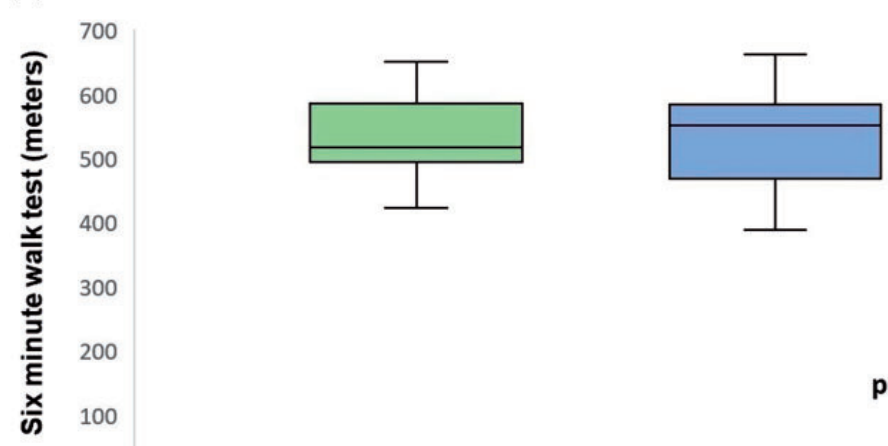

$p=0.711$

Before prehabilitation

After prehabilitation

Figure 2. Changes in functional test results after prehabilitation. (a) Timed Up and Go Test. Before prehabilitation: median, $6.6 \mathrm{~s}$ (interquartile range, 5.6-7.I s). After prehabilitation: median, $6.6 \mathrm{~s}$ (interquartile range, $6.0-7.1 \mathrm{~s}$ ). (b) Six-Minute Walk Test. Before prehabilitation: median, $516 \mathrm{~m}$ (interquartile range, $498-576 \mathrm{~m}$ ). After prehabilitation: median, $550 \mathrm{~m}$ (interquartile range, $482-58 \mathrm{Im}$ ).

important proportion of patients. Results regarding adherence to prehabilitation programs are conflicting, possibly because of differences in the number of staff members involved and their commitment. In an RCT of prehabilitation before liver resection, $95 \%$ of patients completed all exercise sessions. ${ }^{14}$ In contrast, another RCT of prehabilitation in patients undergoing colorectal surgery showed poor adherence to the intense exercise program $(16 \%)$, which probably contributed to its lack of overall efficacy. ${ }^{35}$ Possible causes of this low adherence include lack of social support from family and friends and the patient's low belief in the benefits of fitness. A feasibility study of prehabilitation in patients undergoing colorectal surgery showed moderate adherence; only $50 \%$ of patients completed all training sessions, although missed sessions were known in advance and mainly resulted from unavoidable personal events. $^{15}$

Research has also indicated that supervision of exercise and an interval-based nature of the training program may 


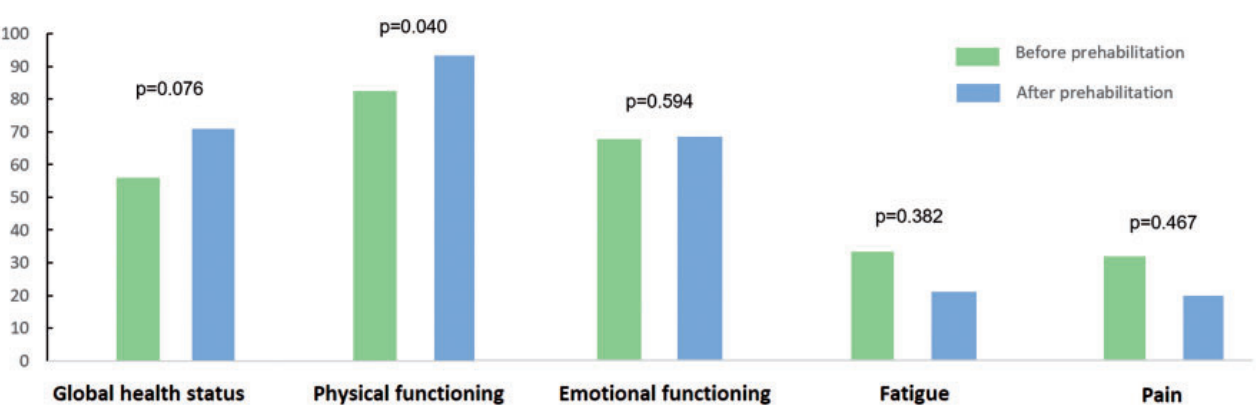

Figure 3. Changes in quality of life (European Organization for Research and Treatment of Cancer Quality of Life Questionnaire (EORTC QLQ-C30)) after prehabilitation. The scores range from 0 to 100 after linear transformation of the raw scores. A high score for a functional scale represents a high level of functioning (healthier), whereas a high score for a symptom scale represents a high level of symptoms/problems.

improve adherence, with interval training being more enjoyable than constant load programs. ${ }^{14,36}$ Variation in exercise supervision may also influence compliance levels. ${ }^{37}$ Importantly, three patients in the present study were excluded because of adverse events during CPET, but without shortterm repercussions. It is crucial to report such adverse events because the risk/benefit balance must be in favor of the patient in future prehabilitation programs. However, no previous study reported adverse outcomes during exercise intervention, which seems surprising in view of some intense exercise programs offered to all-comers, including patients with cardiac comorbidities. Further monitoring of compliance and adverse effects of prehabilitation is required in future prospective research on prehabilitation.

Several limitations of the present feasibility study need to be addressed. First, non-randomized convenience and consecutive sampling may represent a potential source of sampling bias and may limit generalization to the wider population because more health-conscious individuals may choose to participate in studies assessing exercise capacity. No control group was used, and the changes observed in the study could therefore be related to other factors. In view of the small sample size, noncompliant patients were not compared with patients who performed prehabilitation in terms of demographics and clinical outcomes. Missing data $(<10 \%)$ and the lack of adjustment for multiple comparisons may have induce bias in the estimation of parameters, thus limiting the representativeness of the samples. The inclusion of qualitative aspects focusing on patient and care provider perspectives on the approach and delivery of the prehabilitation program would have been beneficial and should be planned in future research. Specific feasibility issues faced by the participants due to HIITtype exercise sessions during prehabilitation for major abdominal surgery would be of great interest for planning of such studies to improve recruitment and adherence. In addition, the number of dedicated staff to inform, motivate, and supervise the patients are factors to be assessed. Finally, this pilot study must be considered a requisite initial step in exploring a prehabilitation program. The delivery of meaningful data is thus limited, and the results should be interpreted with caution. However, the findings of this study can 
inform feasibility and identify modifications needed in the design of a larger, ensuing hypothesis-testing study.

\section{Conclusion}

The implementation of a prehabilitation program before major abdominal surgery is not as easy as it seems in theory. Although data on prehabilitation suggest positive effects on physical fitness, major barriers to implementation of such a program may include insufficient time prior to surgery as well as lack of availability and commitment from surgical staff and patients. Thus, the prehabilitation program described in this report is not feasible in its current form. Sufficient resources are needed to successfully implement and maintain a prehabilitation program for all-comers. Future efforts should focus on identification of those patients who are likely to be eligible and benefit the most and the creation of a dedicated prehabilitation team.

\section{Declaration of conflicting interest}

The authors declare that there is no conflict of interest.

\section{Funding}

This research received no specific grant from any funding agency in the public, commercial, or not-for-profit sectors.

\section{ORCID iDs}

David Martin (D) https://orcid.org/0000-00031585-5231

Basile Pache (D) https://orcid.org/0000-00030969-7345

Bengt Kayser (D) https://orcid.org/0000-00029776-7501

\section{References}

1. Arozullah AM, Khuri SF, Henderson WG, et al. Development and validation of a multifactorial risk index for predicting postoperative pneumonia after major noncardiac surgery. Ann Intern Med 2001; 135: 847-857.

2. Smetana GW, Cohn SL and Lawrence VA. Update in perioperative medicine. Ann Intern Med 2004; 140: 452-461.

3. Carli F and Mayo N. Measuring the outcome of surgical procedures: what are the challenges? Br J Anaesth 2001; 87: 531-533.

4. Lemanu DP, Singh PP, MacCormick AD, et al. Effect of preoperative exercise on cardiorespiratory function and recovery after surgery: a systematic review. World J Surg 2013; 37: 711-720. DOI: 10.1007/s00268012-1886-4.

5. Topp R, Ditmyer M, King K, et al. The effect of bed rest and potential of prehabilitation on patients in the intensive care unit. AACN Clin Issues 2002; 13: 263-276.

6. Bobbio A, Chetta A, Ampollini L, et al. Preoperative pulmonary rehabilitation in patients undergoing lung resection for nonsmall cell lung cancer. Eur J Cardiothorac Surg 2008; 33: 95-98. DOI: 10.1016/j. ejcts.2007.10.003.

7. Jones LW, Peddle CJ, Eves ND, et al. Effects of presurgical exercise training on cardiorespiratory fitness among patients undergoing thoracic surgery for malignant lung lesions. Cancer 2007; 110: 590-598. DOI: $10.1002 /$ cncr. 22830 .

8. Valkenet K, Van De Port IG, Dronkers JJ, et al. The effects of preoperative exercise therapy on postoperative outcome: a systematic review. Clin Rehabil 2011; 25: 99-111. DOI: $10.1177 / 0269215510380830$.

9. Pouwels S, Stokmans RA, Willigendael EM, et al. Preoperative exercise therapy for elective major abdominal surgery: a systematic review. Int J Surg 2014; 12: 134-140. DOI: 10.1016/j.ijsu.2013.11.018.

10. Barberan-Garcia A, Ubre M, Roca J, et al. Personalised prehabilitation in high-risk patients undergoing elective major abdominal surgery: a randomized blinded controlled trial. Ann Surg 2018; 267: 50-56. DOI: $10.1097 /$ sla.0000000000002293.

11. West MA, Loughney L, Lythgoe D, et al. Effect of prehabilitation on objectively measured physical fitness after neoadjuvant treatment in preoperative rectal cancer 
patients: a blinded interventional pilot study. $\mathrm{Br} J$ Anaesth 2015; 114: 244-251. DOI: $10.1093 /$ bja/aeu318.

12. Kothmann E, Batterham AM, Owen SJ, et al. Effect of short-term exercise training on aerobic fitness in patients with abdominal aortic aneurysms: a pilot study. Br J Anaesth 2009; 103: 505-510. DOI: $10.1093 / \mathrm{bja} /$ aep205.

13. Peddle CJ, Jones LW, Eves ND, et al. Effects of presurgical exercise training on quality of life in patients undergoing lung resection for suspected malignancy: a pilot study. Cancer Nurs 2009; 32: 158-165. DOI: $10.1097 /$ NCC.0b013e3181982ca1.

14. Dunne DF, Jack S, Jones RP, et al. Randomized clinical trial of prehabilitation before planned liver resection. $B r J$ Surg 2016; 103: 504-512. DOI: 10.1002/bjs.10096.

15. Northgraves MJ, Arunachalam L, Madden LA, et al. Feasibility of a novel exercise prehabilitation programme in patients scheduled for elective colorectal surgery: a feasibility randomised controlled trial. Support Care Cancer 2020; 28: 3197-3206. DOI: 10.1007/s00520-019-05098-0.

16. Bhatia C and Kayser B. Preoperative highintensity interval training is effective and safe in deconditioned patients with lung cancer: a randomized clinical trial. $J$ Rehabil Med 2019; 51: 712-718. DOI: 10.2340/16501977-2592.

17. Campeau L. Letter: Grading of angina pectoris. Circulation 1976; 54: 522-523.

18. The Criteria Committee of the New York Heart Association. Nomenclature and criteria for diagnosis of diseases of the heart and great vessels. 9th ed. Boston: Little, Brown and Company, 1994, p.253-256.

19. Vestbo J, Hurd SS, Agusti AG, et al. Global strategy for the diagnosis, management, and prevention of chronic obstructive pulmonary disease: GOLD executive summary. Am $J$ Respir Crit Care Med 2013; 187: 347-365. DOI: $10.1164 /$ rccm.201204-0596PP.

20. Melloul E, Lassen K, Roulin D, et al. Guidelines for perioperative care for pancreatoduodenectomy: Enhanced Recovery After Surgery (ERAS) recommendations 2019. World J Surg 2020; 44: 2056-2084. DOI: $10.1007 / \mathrm{s} 00268-020-05462-w$.
21. Gustafsson UO, Scott MJ, Hubner M, et al. Guidelines for perioperative care in elective colorectal surgery: Enhanced Recovery After Surgery (ERAS $\left.\left({ }^{\circledR}\right)\right)$ Society recommendations: 2018. World J Surg 2019; 43: 659-695. DOI: 10.1007/s00268-018-4844-y.

22. Melloul E, Hübner $M, \operatorname{Scott} M$, et al. Guidelines for perioperative care for liver surgery: Enhanced Recovery After Surgery (ERAS) Society recommendations. World $J$ Surg 2016; 40: 2425-2440. DOI: 10.1007/ s00268-016-3700-1.

23. Steffens D, Young J, Beckenkamp PR, et al. Feasibility and acceptability of a preoperative exercise program for patients undergoing major cancer surgery: results from a pilot randomized controlled trial. Pilot Feasibility Stud 2021; 7: 27. DOI: 10.1186/s40814-02100765-8.

24. Slankamenac K, Graf R, Barkun J, et al. The comprehensive complication index: a novel continuous scale to measure surgical morbidity. Ann Surg 2013; 258: 1-7. DOI: 10.1097/SLA.0b013e318296c732.

25. Dindo D, Demartines $\mathrm{N}$ and Clavien PA. Classification of surgical complications: a new proposal with evaluation in a cohort of 6336 patients and results of a survey. Ann Surg 2004; 240: 205-213.

26. EORTC Quality of Life Group, version 3, http://groups.eortc.be/qol/

27. Dunne DF, Jones RP, Lythgoe DT, et al. Cardiopulmonary exercise testing before liver surgery. J Surg Oncol 2014; 110: 439-444. DOI: $10.1002 /$ jso. 23670.

28. Martin D, Romain B, Pache B, et al. Physical activity and outcomes in colorectal surgery: a pilot prospective cohort study. Eur Surg Res 2020; 61: 23-33. DOI: $10.1159 / 000507578$.

29. Aaronson NK, Ahmedzai S, Bergman B, et al. The European Organization for Research and Treatment of Cancer QLQC30: a quality-of-life instrument for use in international clinical trials in oncology. $J$ Natl Cancer Inst 1993; 85: 365-376. DOI: 10.1093/jnci/85.5.365.

30. Pompili C, Koller M, Velikova G, et al. EORTC QLQ-C30 summary score reliably detects changes in QoL three months after anatomic lung resection for non-small 
cell lung cancer (NSCLC). Lung Cancer 2018; 123: 149-154. DOI: $10.1016 / \mathrm{j}$. lungcan.2018.07.021.

31. Von Elm E, Altman DG, Egger M, et al. The Strengthening the Reporting of Observational Studies in Epidemiology (STROBE) statement: guidelines for reporting observational studies. Ann Intern Med 2007; 147: 573-577. DOI: 10.7326/00034819-147-8-200710160-00010.

32. NHS, Interim Management and Support, Delivering cancer waiting times - a good practice guide. https://www.england.nhs.uk/ wp-content/uploads/2015/03/deliveringcancer-wait-times.pdf

33. Bolshinsky V, Li MH, Ismail $\mathrm{H}$, et al. Multimodal prehabilitation programs as a bundle of care in gastrointestinal cancer surgery: a systematic review. Dis Colon Rectum 2018; 61: 124-138. DOI: 10.1097/dcr.000 0000000000987.

34. Luther A, Gabriel J, Watson RP, et al. The impact of total body prehabilitation on post- operative outcomes after major abdominal surgery: a systematic review. World J Surg 2018; 42: 2781-2791. DOI: 10.1007/s00268018-4569-y.

35. Carli F, Charlebois $\mathrm{P}$, Stein $\mathrm{B}$, et al. Randomized clinical trial of prehabilitation in colorectal surgery. Br J Surg 2010; 97: 1187-1197. DOI: 10.1002/bjs.7102.

36. Bartlett JD, Close GL, MacLaren DP, et al. High-intensity interval running is perceived to be more enjoyable than moderateintensity continuous exercise: implications for exercise adherence. J Sports Sci 2011; 29: 547-553. DOI: 10.1080/02640414.2010. 545427.

37. Hughes MJ, Hackney RJ, Lamb PJ, et al. Prehabilitation before major abdominal surgery: a systematic review and meta-analysis. World J Surg 2019; 43: 1661-1668. DOI: 10.1007/s00268-019-04950-y. 